\title{
Cause-specific child and adolescent mortality in the UK and EU15+ countries
}

\author{
Joseph Lloyd Ward (1) , ${ }^{1}$ Ingrid Wolfe, ${ }^{2}$ Russell M Viner (i) ${ }^{1}$
}

- Additional material is published online only. To view please visit the journal online (http://dx.doi.org/10.1136/ archdischild-2019-318097).

'Population, Policy and Practice Research and Teaching Department, UCL Great Ormond Street Institute of Child Health, London, UK

${ }^{2}$ Department of Women and Children's Health, King's College London, London, UK

\section{Correspondence to} Dr Joseph Lloyd Ward, University College London Institute of Child Health, London, London, UK; joseph.ward@ucl.ac.uk

Received 16 August 2019 Revised 14 April 2020 Accepted 23 May 2020 Published Online First 26 August 2020

\section{Check for updates}

(c) Author(s) (or their employer(s)) 2020. No commercial re-use. See rights and permissions. Published by BMJ.

To cite: Ward IL, Wolfe I, Viner RM. Arch Dis Child 2020;105:1055-1060.

\section{ABSTRACT}

Objective To compare cause-specific UK mortality in children and young people (CYP) with EU15+ countries (European Union countries pre-2004, Australia, Canada and Norway).

Design Mortality estimates were coded from the WHO World Mortality Database. Causes of death were mapped using the Global Burden of Disease mortality hierarchy to 22 cause groups. We compared UK mortality by cause, age group and sex with EU15+ countries in 2015 (or latest available) using Poisson regression models. We then ranked the UK compared with the EU15+ for each cause.

Setting The UK and EU15+ countries.

Participants CYP aged 1-19.

Main outcome measure Mortality rate per 100000 and number of deaths.

Results UK mortality in 2015 was significantly higher than the EU15+ for common infections (both sexes aged $1-9$, boys aged 10-14 and girls aged 15-19); chronic respiratory conditions (both sexes aged 5-14); and digestive, neurological and diabetes/urological/blood/ endocrine conditions (girls aged 15-19). UK mortality was significantly lower for transport injuries (boys aged 15-19). The UK had the worst to third worst mortality rank for common infections in both sexes and all age groups, and in five out of eight non-communicable disease (NCD) causes in both sexes in at least one age group. UK mortality rank for injuries in 2015 was in the top half of countries for most causes.

Conclusions UK CYP mortality is higher than a group of comparable countries for common infections and multiple NCD causes. Excess UK CYP mortality may be amenable to health system strengthening.

\section{INTRODUCTION}

UK mortality outcomes for children and young people (CYP) are worse than in comparable highincome countries. ${ }^{1-3}$ In 2014 we estimated excess UK mortality to result in around 1000 additional deaths in infants and young children and 300 extra deaths in adolescents and young adults (10-24) each year, ${ }^{4}$ when compared with the median mortality in a group of similar countries (the EU15+: European Union countries pre-2004, Norway, Australia and Canada). Our recent updated analysis demonstrated continued poor UK outcomes for all-cause and non-communicable diseases (NCD), particularly in younger children. ${ }^{5}$ Slower rates of decline in mortality in the UK will mean these differences are likely to widen; we estimated infant and 1-4 all-cause mortality could be $180 \%$ and $140 \%$ of the EU15 + median by 2030, if current trends

\section{What is already known on this topic?}

- UK all-cause mortality outcomes for children and young people (CYP) are worse than in similar high-income nations.

- Little is known about comparative differences in cause-specific mortality.

\section{What this study adds?}

Although UK CYP mortality continues to decline, CYP mortality for common infections and multiple non-communicable disease causes is higher than a comparable group of high-income countries.

- The UK continues to have lower injury mortality than most EU15+ countries.

- Conditions where there is excess CYP mortality in the UK include those which may be amenable to health system strengthening.

continue. ${ }^{5}$ Comparisons between the UK and countries with the lowest CYP mortality rates in Europe are particularly stark. In 2014 Wolfe et $a l^{2}$ showed that reducing UK 0-14 mortality to the rate seen in Sweden would save 2000 lives a year, and Zylbersztejn $e t$ al recently estimated there to be around 600 extra deaths in children aged 0-4 years old from all causes in England compared with Sweden. ${ }^{6}$

Understanding differences in cause-specific mortality in CYP in the UK is essential for designing effective solutions to improve survival. Yet the most recent systematic international comparative analysis of mortality by cause used data which are now a decade old. ${ }^{4}$ To better understand the UK's continued poor performance, and so inform potential policy interventions to improve outcomes, a detailed assessment of mortality by cause group across the early life course is required.

This study aims to compare child and adolescent mortality outcomes by cause in the UK with the EU15 +, a group of comparable high-income countries with similar healthcare systems and disease patterns used in previous benchmarking exercises. ${ }^{4}$ We limited our analysis to CYP aged 1-19 years old as international comparisons of infant mortality are problematic due to differences in the classification of deaths, causes of death are distinct from those in older groups, and the UK's position within Europe has recently been described. ${ }^{7}$ We used the methods described in the recent Global Burden of Disease (GBD) study to categorise causes of death 
into 22 cause groups and redistribute inappropriately coded deaths ('garbage codes'). ${ }^{8}$ We then ranked the UK relative to the EU15+ for mortality rate by cause, sex and age group.

\section{METHODS}

\section{Data}

National mortality estimates for the UK and EU15 + countries (Austria, Belgium, Denmark, Finland, France, Germany, Greece, Ireland, Italy, Luxembourg, The Netherlands, Portugal, Spain, Sweden, Australia, Canada and Norway), available by year, sex and 5-year group (1-4, 5-9, 10-14 and 15-19), were accessed from the WHO World Mortality Database (WMD) on 9 October 2017 (http://www.who.int/healthinfo/mortality_data/en/). We limited this analysis to country-years using International Classification of Diseases 10 (ICD-10) codes to improve comparability between countries and over time. Estimates were available to 2015 for the UK and all other countries except Belgium, France, Greece, Ireland, Italy and Portugal (2014) and Canada (2013).

We used the GBD mortality hierarchy to map ICD-10 coded cause of death to cause groups. The GBD classifies deaths across four levels. For example, acute lymphoblastic leukaemia (level 4 ) is classified within leukaemias (level 3), neoplasms (level 2) and NCD (level 1). We mapped causes in the WMD to 3 level 1 categories (communicable, maternal, neonatal and nutritional diseases; NCD; injuries) and 21 level 2 categories (7 communicable disease causes; 10 NCD causes; 4 injury causes). We then separated the level 2 cause 'self-harm and interpersonal violence' into self-harm and violence, as these causes of death have different policy responses.

A significant proportion of deaths within the WMD were assigned ambiguous or inappropriate underlying causes. We used a minimally modified version of the GBD methodology for redistributing these 'garbage codes' to each level 2 cause group. We first classified garbage codes (levels 1-4) as described by the GBD 2016, and then proportionately redistributed deaths within each level for all causes, avoiding the need for detailed target cause lists for specific codes as used in the GBD. ${ }^{9}$

\section{Procedures}

To account for the large annual variation in mortality and low numbers of deaths for some causes, we first calculated 3-year lagged mean number of deaths and mortality rate per 100000 for each cause, age group, sex and country-year available. We then excluded causes contributing less than $1 \%$ of the total UK mortality burden within each age and sex group. We examined current differences in mortality between the UK and EU15+ using Poisson regression models. We calculated incidence rate ratio (IRR) with $95 \% \mathrm{CI}$ to compare the 3-year lagged mean number of deaths by cause in the UK with all 17 EU15+ countries in 2015 among children aged 1-4, 5-9, 10-14 and 15-19 years old. If countries did not have data for 2015, we used the latest available data year. We were unable to use lagged mean estimates for Greece as data were only available for 2014. We used midyear population denominators provided by the WMD as an offset in each model (meaned over the same 3-year period as number of deaths). Where population data were not available from the WMD, we used estimates provided by national statistics databases. As patterns of cause of death are similar for both sexes during early childhood (1-9), but with large differences during adolescence, we present results for both sexes for children aged 1-4 and 5-9 years old, and by sex for adolescents aged 10-14 and 15-19 years old.
To account for multiple comparisons, we defined significant differences in mortality between the UK and EU15 + as p $<0.005$. This approximates to a modified Bonferroni correction for 10 comparisons per age group/sex but avoids using different significance thresholds for each group. We then ranked the UK compared with EU15 + countries using the 3-year lagged mean mortality rate per 100000 in 2015 (or latest date available). We then performed a sensitivity analysis repeating the procedures described above but excluding all garbage coded deaths. All analyses were performed in Stata V.14.

\section{RESULTS}

The 3-year lagged mean number of deaths in CYP aged 1-19 in 2015 in the UK was 2110 (1281 boys and 829 girls), and the 3 -year lagged all-cause mortality rate was 14.6 per 100000 . Of the deaths, $44.8 \%$ were among $15-19$ year olds, $15.7 \%$ were among $10-14$ year olds, $14.6 \%$ were among 5-9year olds and $24.9 \%$ were among 1-4 year olds. Cause of death was inappropriately assigned and redistributed for 613 deaths (29.1\%).

Tables 1-3 show the 3-year lagged mean annual number of deaths in the UK in 2015 and the proportion of total mortality for children aged 1-4 and 5-9 years old (both sexes) and for children aged 10-14 and 15-19 years old by sex. The IRR for UK mortality compared with EU15 + countries is also shown, with $95 \%$ CI. Significant $(p<0.005)$ differences in mortality are highlighted in red.

The UK had significantly higher mortality for common infections in children aged 1-4 and 5-9 years old (both sexes), boys aged 10-14 and girls aged 15-19, and for chronic respiratory conditions in children aged 5-9 and 10-14 years old in both sexes. Among girls aged 15-19, the UK also had significantly higher mortality than the EU15 + for digestive disorders, neurological disorders and diabetes/urological/blood/endocrine disorders (DUBE). UK mortality was significantly lower than the EU15 + for transport injuries among boys aged 15-19.

Table 4 shows the most recent UK mortality rank by age group and sex compared with the other EU15 + countries using the 3-year lagged mortality rate per 100000 in 2015 (or latest available date) for each country. In order to demonstrate the UK's position relative to other EU15 + countries, cells in this table are shaded green (lowest mortality) to yellow (median mortality), to red (highest mortality). The UK ranked 17th or 18th (highest or second highest mortality) for childhood infections across all age groups in boys and between 16th and 18th for girls. The UK ranked 15th-18th for chronic respiratory diseases in boys and girls in all age groups, and in the bottom five countries for most other NCD mortality groups except neoplasms. All causes of injury mortality in the UK were ranked in the top 10 countries in boys and girls for all age groups except transport injuries in boys aged 10-14 (11th) and self-harm in boys aged 15-19 (11th).

Results from the sensitivity analyses showed a similar pattern of mortality differences between the UK and EU15+. After excluding redistributed deaths, the UK still had significantly higher mortality for common infections in children aged 1-4 years old (both sexes), chronic respiratory diseases in children aged 10-14 years old (both sexes) and neurological conditions in girls aged 15-19. The UK had significantly lower mortality than the EU15 + for transport injuries in boys aged 15-19, unintentional injuries in children aged 1-4 (both sexes) and self-harm in girls aged 15-19. The IRR for other age/cause groups identified in the main analysis as having higher mortality in the UK compared with the EU15 + was similar in the sensitivity analysis, but no longer reached significance using the reduced number 
Table 1 IRR with 95\% CI for 3-year lagged mean number of deaths in UK compared with EU15+ in 2015* for children aged 1-4 and 5-9years old (both sexes)

\begin{tabular}{|c|c|c|c|c|c|c|c|c|c|c|c|c|}
\hline & \multicolumn{6}{|c|}{ Both sexes, 1-4 } & \multicolumn{6}{|c|}{ Both sexes, 5-9 } \\
\hline & Deathst & $\%$ of total & IRR & $P$ value & Lower Cl & Upper Cl & Deathst & $\%$ of total & IRR & $P$ value & Lower $\mathrm{Cl}$ & Upper Cl \\
\hline \multicolumn{13}{|l|}{ Communicable/neonatal } \\
\hline Common infections & 85 & 16.2 & 2.08 & $<0.001$ & 1.61 & 2.67 & 25 & 8.0 & 2.38 & $<0.001$ & 1.48 & 3.83 \\
\hline Neonatal disorders & 11 & 2.1 & 1.45 & 0.28 & 0.74 & 2.85 & 3 & 1.0 & 1.12 & 0.86 & 0.32 & 3.90 \\
\hline Other communicable & 7 & 1.3 & 2.27 & 0.07 & 0.92 & 5.56 & & & & & & \\
\hline \multicolumn{13}{|l|}{ NCD } \\
\hline Neoplasms & 93 & 17.8 & 0.94 & 0.58 & 0.75 & 1.17 & 106 & 34.4 & 0.91 & 0.34 & 0.74 & 1.11 \\
\hline Cardiovascular diseases & 23 & 4.4 & 1.22 & 0.40 & 0.77 & 1.91 & 13 & 4.4 & 1.26 & 0.45 & 0.69 & 2.28 \\
\hline Chronic respiratory diseases & 13 & 2.4 & 1.76 & 0.08 & 0.93 & 3.31 & 14 & 4.4 & 3.25 & $<0.001$ & 1.66 & 6.37 \\
\hline Digestive diseases & 11 & 2.0 & 1.85 & 0.09 & 0.91 & 3.74 & 9 & 2.8 & 2.52 & 0.02 & 1.12 & 5.65 \\
\hline Neurological disorders & 50 & 9.5 & 1.23 & 0.20 & 0.90 & 1.67 & 19 & 6.2 & 0.87 & 0.56 & 0.53 & 1.40 \\
\hline DUBE & 52 & 9.9 & 1.01 & 0.94 & 0.75 & 1.36 & 28 & 9.1 & 1.12 & 0.60 & 0.74 & 1.68 \\
\hline Other NCD & 87 & 16.5 & 1.00 & 0.99 & 0.79 & 1.26 & 38 & 12.4 & 1.26 & 0.20 & 0.89 & 1.79 \\
\hline \multicolumn{13}{|l|}{ Injuries } \\
\hline Transport injuries & 23 & 4.4 & 0.60 & 0.02 & 0.39 & 0.93 & 23 & 7.5 & 0.68 & 0.08 & 0.44 & 1.05 \\
\hline Unintentional injuries & 58 & 11.0 & 0.71 & 0.01 & 0.54 & 0.94 & 18 & 6.0 & 0.56 & 0.02 & 0.35 & 0.90 \\
\hline Interpersonal violence & 6 & 1.2 & 0.46 & 0.06 & 0.20 & 1.02 & 4 & 1.4 & 0.53 & 0.21 & 0.20 & 1.44 \\
\hline
\end{tabular}

Causes contributing to less than $1 \%$ of total deaths are not shown.

Causes where there were significant differences in mortality between the UK and EU15+ are highlighted in red.

${ }^{*}$ For EU15+ countries without data to 2015, we used lagged mean number of deaths over the 3 years prior to the latest available data year in Poisson regression models for all countries except Greece, where data were only available for 2014.

tMean annual number of deaths between 2013 and 2015 in the UK rounded to the nearest whole number.

DUBE, diabetes/urological/blood/endocrine disorders; EU15+, European Union countries pre-2004, Norway, Australia and Canada; IRR, incidence rate ratio; NCD, non-communicable disease.

of deaths. There were no substantial differences between UK mortality rank by cause of death compared with EU15 + countries within the sensitivity analysis (see online supplementary material).

\section{DISCUSSION}

We found CYP mortality in the UK to be significantly higher than the EU15 + for multiple causes across all age groups and both sexes. If the UK were to reduce its mortality rate in these conditions to the
EU15 + median, around 145 deaths would be avoided each year. The UK also ranked between highest or third highest for mortality from common infections across age groups and ranked poorly for most NCD causes, particularly among adolescents.

\section{Comparison with the literature}

Previous studies have shown a significant proportion of excess UK mortality to result from poor outcomes in younger children. $^{2610}$ The results shown here build on this evidence, but further

\begin{tabular}{|c|c|c|c|c|c|c|c|c|c|c|c|c|}
\hline & \multicolumn{6}{|c|}{ Boys 10-14 } & \multicolumn{6}{|c|}{ Girls 10-14 } \\
\hline & Deathst & $\%$ of total & IRR & $P$ value & Lower Cl & Upper CI & Deathst & $\%$ of total & IRR & $P$ value & Lower CI & Upper CI \\
\hline \multicolumn{13}{|l|}{ Communicable/maternal } \\
\hline Common infections & 12 & 6.3 & 3.02 & $<0.001$ & 1.51 & 6.06 & 6 & 4.6 & 1.75 & 0.21 & 0.73 & 4.21 \\
\hline \multicolumn{13}{|l|}{ NCD } \\
\hline Chronic respiratory diseases & 11 & 5.7 & 3.65 & $<0.001$ & 1.72 & 7.77 & 9 & 6.7 & 4.29 & $<0.001$ & 1.85 & 9.95 \\
\hline Digestive diseases & 3 & 1.6 & 2.45 & 0.18 & 0.66 & 9.15 & 4 & 3.2 & 3.87 & 0.03 & 1.17 & 12.77 \\
\hline Neurological disorders & 13 & 6.7 & 1.13 & 0.69 & 0.62 & 2.05 & 12 & 8.2 & 1.26 & 0.47 & 0.67 & 2.38 \\
\hline DUBE & 14 & 7.5 & 1.25 & 0.45 & 0.70 & 2.20 & 15 & 10.9 & 1.34 & 0.31 & 0.77 & 2.32 \\
\hline Other NCD & 15 & 7.8 & 1.03 & 0.91 & 0.59 & 1.79 & 16 & 11.7 & 1.12 & 0.68 & 0.66 & 1.89 \\
\hline Interpersonal violence & 2 & 1.0 & 0.61 & 0.52 & 0.14 & 2.74 & 2 & 1.2 & 0.51 & 0.41 & 0.10 & 2.54 \\
\hline
\end{tabular}

Causes contributing to less than $1 \%$ of total deaths are not shown.

Causes where there were significant differences in mortality between the UK and EU15+ are highlighted in red.

${ }^{*}$ For EU15+ countries without data to 2015, we used lagged mean number of deaths over the 3 years prior to the latest available data year in Poisson regression models for all countries except Greece, where data were only available for 2014.

†Mean annual number of deaths between 2013 and 2015 in the UK rounded to the nearest whole number.

DUBE, diabetes/urological/blood/endocrine disorders; EU15+, European Union countries pre-2004, Norway, Australia and Canada; IRR, incidence rate ratio; NCD, non-communicable disease. 
Table 3 IRR with 95\% CI for 3-year lagged mean number of deaths in UK compared with EU15+ in 2015* for children aged 15-19years old by sex

\begin{tabular}{|c|c|c|c|c|c|c|c|c|c|c|c|c|}
\hline & \multicolumn{6}{|c|}{ Boys 15-19 } & \multicolumn{6}{|c|}{ Girls 15-19 } \\
\hline & Deathst & $\%$ of total & IRR & $P$ value & Lower $\mathrm{Cl}$ & Upper Cl & Deathst & $\%$ of total & IRR & $P$ value & Lower $\mathrm{Cl}$ & Upper CI \\
\hline \multicolumn{13}{|l|}{ Communicable/maternal } \\
\hline Common infections & 15 & 2.5 & 2.14 & 0.01 & 1.19 & 3.86 & 17 & 5.3 & 2.91 & $<0.001$ & 1.62 & 5.21 \\
\hline \multicolumn{13}{|l|}{ NCD } \\
\hline Neoplasms & 81 & 13.1 & 0.94 & 0.60 & 0.74 & 1.19 & 63 & 19.6 & 1.04 & 0.75 & 0.80 & 1.37 \\
\hline Cardiovascular diseases & 29 & 4.7 & 1.18 & 0.43 & 0.79 & 1.75 & 16 & 5.0 & 1.18 & 0.54 & 0.69 & 2.02 \\
\hline Chronic respiratory diseases & 8 & 1.3 & 1.54 & 0.28 & 0.70 & 3.37 & 8 & 2.6 & 2.68 & 0.02 & 1.17 & 6.09 \\
\hline Digestive diseases & 7 & 1.1 & 2.19 & 0.08 & 0.90 & 5.32 & 8 & 2.6 & 3.60 & 0.0041 & 1.50 & 8.62 \\
\hline Neurological disorders & 43 & 6.9 & 1.60 & 0.01 & 1.14 & 2.25 & 27 & 8.4 & 2.02 & 0.002 & 1.30 & 3.12 \\
\hline DUBE & 30 & 4.7 & 1.74 & 0.01 & 1.15 & 2.62 & 30 & 9.4 & 1.81 & 0.0045 & 1.20 & 2.72 \\
\hline MSK & & & & & & & 4 & 1.4 & 3.42 & 0.04 & 1.05 & 11.12 \\
\hline Other NCD & 16 & 2.6 & 0.85 & 0.54 & 0.50 & 1.43 & 18 & 5.5 & 1.15 & 0.58 & 0.69 & 1.93 \\
\hline \multicolumn{13}{|l|}{ Injuries } \\
\hline Transport injuries & 152 & 24.4 & 0.70 & $<0.001$ & 0.59 & 0.83 & 57 & 17.8 & 0.77 & 0.07 & 0.59 & 1.02 \\
\hline Unintentional injuries & 53 & 8.5 & 0.92 & 0.56 & 0.69 & 1.23 & 13 & 4.1 & 1.05 & 0.87 & 0.59 & 1.89 \\
\hline Self-harm & 168 & 27.0 & 1.05 & 0.55 & 0.89 & 1.24 & 51 & 15.7 & 0.70 & 0.02 & 0.52 & 0.94 \\
\hline Interpersonal violence & 8 & 1.2 & 0.46 & 0.04 & 0.22 & 0.96 & & & & & & \\
\hline
\end{tabular}

Causes contributing to less than $1 \%$ of total deaths are not shown.

Causes where there were significant differences in mortality between the UK and EU15+ are highlighted in red.

${ }^{*}$ For EU15+ countries without data to 2015, we used lagged mean number of deaths over the 3 years prior to the latest available data year in Poisson regression models for all countries except Greece, where data were only available for 2014.

tMean annual number of deaths between 2013 and 2015 in the UK rounded to the nearest whole number.

DUBE, diabetes/urological/blood/endocrine disorders; EU15+, European Union countries pre-2004, Norway, Australia and Canada; IRR, incidence rate ratio; MSK, musculoskeletal disorders; NCD, non-communicable disease.

demonstrate stark differences in outcomes for older children and adolescents, ${ }^{5}$ particularly among girls. UK mortality for girls aged 15-19 was found to be significantly higher than EU15 + countries for conditions accounting for around a quarter of total deaths in this age group. Adolescent girls in the UK also ranked 17th or 18th in five out of the eight NCD groups. Worryingly, total mortality has actually increased year on year since 2013 among adolescent girls aged 15-19 in the UK, and is plateauing among adolescent boys, in contrast to the EU15 + median, which is continuing to improve (data not shown).

NCD causes appear to be a major contributor to higher than expected CYP mortality in the UK. ${ }^{4510}{ }^{11}$ Similar to our findings,

Table 4 UK rank for 3-year lagged mean mortality rate per 100000 compared with 17 EU15+ countries in 2015*

\begin{tabular}{|c|c|c|c|c|c|c|}
\hline & $1-4$ & $5-9$ & $10-14$ & $10-14$ & $15-19$ & $15-19$ \\
\hline & Both sexes & Both sexes & Boys & Girls & Boys & Girls \\
\hline \multicolumn{7}{|l|}{ Communicable/maternal } \\
\hline Common infections & 17 & 18 & 17 & 16 & 18 & 18 \\
\hline Neonatal disorders & 15 & 11 & & & & \\
\hline Other communicable & 15 & & & & & \\
\hline \multicolumn{7}{|l|}{ NCD } \\
\hline Neoplasms & 12 & 6 & 7 & 6 & 9 & 14 \\
\hline Cardiovascular diseases & 16 & 15 & 13 & 12 & 15 & 12 \\
\hline Chronic respiratory diseases & 15 & 18 & 18 & 18 & 16 & 18 \\
\hline Digestive diseases & 17 & 18 & 17 & 16 & 15 & 18 \\
\hline Neurological disorders & 13 & 7 & 14 & 13 & 17 & 17 \\
\hline DUBE & 13 & 10 & 14 & 14 & 17 & 17 \\
\hline MSK & & & & & & 18 \\
\hline Other NCD & 10 & 15 & 12 & 13 & 7 & 10 \\
\hline \multicolumn{7}{|l|}{ Injuries } \\
\hline Transport injuries & 5 & 5 & 11 & 8 & 7 & 8 \\
\hline Unintentional injuries & 6 & 3 & 9 & 10 & 9 & 10 \\
\hline Self-harm & & & 6 & 7 & 11 & 6 \\
\hline Interpersonal violence & 3 & 6 & 7 & 8 & 6 & \\
\hline
\end{tabular}

Causes contributing to less than $1 \%$ of total deaths are not shown.

Cells are shaded according to UK mortality rank compared with 17 EU15+ countries, from green (1=lowest mortality) to yellow/orange (9.5=median mortality), to red (18=highest mortality). *Rank was calculated using 3-year lagged mean mortality rate per 100000 for 2015 in the UK compared with all EU15+ countries except Greece, where data were only available for 2014 For EU15+ countries without data to 2015, we used lagged mean mortality rate over the 3 years prior to the latest available data year.

DUBE, diabetes/urological/blood/endocrine disorders; EU15+, European Union countries pre-2004, Norway, Australia and Canada; MSK, musculoskeletal disorders; NCD, non-communicable disease. 
Viner $e t a l^{4}$ found the UK to have higher CYP mortality than the $\mathrm{EU} 15+$ for endocrine, respiratory, digestive, and particularly neuropsychiatric causes (epilepsy, cerebral palsy and substance misuse). In our analysis, UK mortality for neurological causes (predominantly epilepsy) was significantly higher among adolescent girls, with around twice the number of deaths in the UK compared with the EU15+ average. The UK also ranked 17th out of 18 countries for neurological conditions among adolescents aged 15-19years old in both sexes. UK outcomes from chronic respiratory conditions, of which the majority were deaths due to asthma, were significantly worse among boys and girls aged 5-14. UK mortality for asthma in CYP has previously been shown to be up to 11 times higher than comparable countries, ${ }^{11}$ and these differences persist after adjustment for variation in prevalence. ${ }^{12}$ Although we also found mortality for digestive conditions and DUBE to be significantly higher in the UK than in EU15+ in girls aged 15-19, both of these cause groups contain a wide range of level 3 causes, and so these results are difficult to interpret.

These findings also highlight the contribution of communicable diseases to excess mortality among CYP. Similar to our findings, Tambe $e$ t $a l^{1011}$ found higher CYP mortality rates for infections in the UK compared with Sweden and others have shown poor UK outcomes and slow rates of decline for childhood infections such as pneumonia and meningococcal disease. ${ }^{12-15}$ We found the UK to have significantly higher mortality for common infections than the EU15 + in children aged 1-9years old (both sexes), boys aged 10-14 and girls aged 15-19, and ranked in the bottom three countries across all age groups and both sexes. Although common infections account for a relatively small proportion of total CYP deaths, reducing UK mortality to the EU15 + median would save around 85 lives a year in those aged 1-19 years old(see online supplementary material).

The UK has historically performed well in injury mortality compared with other high-income nations. ${ }^{4}$ Our findings support this, showing significantly lower mortality for transport injuries among boys aged $15-19$, which account for around $21 \%$ of all injury-related deaths in those aged 1-19. We also found the UK mortality rank for most causes of death due to injury to be in the top half of countries for all age groups.

\section{Strengths and weaknesses}

We used high-quality mortality estimates provided by the WHO WMD and compared outcomes with a group of countries similar to the UK (the EU15+) used in previous benchmarking exercises. $^{3-5} 1216$ To improve comparability between countries and over time, we only included deaths coded to ICD-10, and we used the mean number of deaths and mortality rates over a 3 -year period to account for large annual variability in mortality for some causes. All countries had data available within 1 year of the latest UK data release (2015), except Canada where data were available to 2013.

Differences in the way countries compile mortality statistics and whether deaths are reported by registration year or death year may have affected our results, particularly towards the end of the study period. ${ }^{17}$ The use of broad GBD level 2 cause groups reduces the risk of coding differences between countries and over time, but also has limitations due to the large number of conditions included in some groups. We considered outcomes to be significantly different if $\mathrm{p}<0.005$ to account for multiple testing. We used this threshold to approximate a Bonferroni correction for 10 comparisons but maintain consistency across all age/sex groups. However, our results may have differed using alternative approaches and we may have underestimated UK excess mortality, particularly in adolescent boys. The IRRs for the number of deaths from common infections, neurological conditions and DUBE were all higher in the UK than in EU15 + among boys aged 15-19, with $\mathrm{p}<0.05$ but $\mathrm{p}>0.005$.

Using alternative methods to redistribute deaths may have affected our results. However, our sensitivity analysis using only appropriately coded deaths found the same pattern of mortality differences as the main analysis, with the UK having higher mortality for common infections, neurological and respiratory conditions, and good outcomes for injuries. Our results may have also been influenced by other errors in death certification. Deaths due to self-harm are known to be under-reported, ${ }^{18}$ and mortality due to common infections may be over-reported, with a large proportion potentially attributable to underlying medical conditions. ${ }^{21419}$ However, as our results show excess UK mortality across a range of conditions in all age groups, they are unlikely to be fully explained by differences in coding alone.

\section{Meaning, mechanisms and policy responses}

This study aimed to identify cause-specific differences in CYP mortality in the UK compared with the EU15+. In order to explain why the UK performs poorly, we should consider the many determinants of child health and survival, how these differ between the UK and comparable countries, and what policy responses are needed to improve outcomes.

At the macro level, the social and economic environments in which CYP live are likely to be important contributors to poor health outcomes in the UK. ${ }^{1-3}{ }^{20}$ A recent study found that characteristics at birth and socioeconomic factors explain over $80 \%$ of the excess all-cause mortality among children 2 days- 4 years in England compared with Sweden. ${ }^{6}$ Risk factors for CYP mortality such as prematurity and low birth weight, which are strongly associated with social deprivation, are also higher in the UK than many other EU15 + countries. ${ }^{21}$ UK relative child poverty levels are higher than the best performing EU15 + countries for child mortality, and levels are predicted to increase. ${ }^{22}$ In response, the Royal College of Paediatrics and Child Health has argued that in addition to direct fiscal measures, actions to reduce the impact of poverty on child health outcomes should include a cross-government child health strategy for the UK, and developing a child-health-in-all-policies approach to reduce health and educational inequities. ${ }^{1}$ Many of the intermediate determinants of UK CYP survival are shaped by local government, and so recent reductions in funding levels, in particular spending on public health, risk impeding progress in improving mortality. ${ }^{23}{ }^{24}$ At the individual level, personal, family and community conditions are strongly affected by the distal factors described above, but some may also be realised through health behaviours. Policy responses to these should be considered in the context of wider social determinants.

Our data show the UK has excess mortality for cause groups which are sensitive to healthcare improvement. For example, it is estimated that there are avoidable factors in two-thirds of UK deaths due to asthma ${ }^{25}$ and a quarter of deaths in children with epilepsy. ${ }^{26}$ Modifiable factors were also identified in $27 \%$ of deaths assessed by child death overview panels in England in 2016/2017, and has been increasing since $2012 .{ }^{27}$ UK mortality rates among children aged 1-14 for causes of death which are thought to be amenable to healthcare also appear to be higher than most countries of the Organisation for Economic Co-operation and Development. ${ }^{28}$

The NHS Long Term Plan in England, ${ }^{29}$ and similar strategies in devolved nations, offer real opportunities to modify the operation of health services to reduce the impact of social deprivation and other social determinants on child health outcomes. Improving coordination between primary and secondary care in the UK, 
which is worse than comparable countries, ${ }^{30}$ also has the potential to improve child health outcomes. ${ }^{31}$ This may include bringing paediatric expertise closer to the front line as the failure to recognise serious illness by health professionals not trained in paediatrics is a recurring factor in childhood deaths. ${ }^{32}$

Focusing on high burden causes would direct efforts to improve child survival where they are most needed. For example, the planned development of paediatric clinical networks for longterm conditions within the NHS Long Term Plan should focus on conditions where the UK has excess mortality. ${ }^{29}$ Investment in data systems relating to child mortality is also needed across the UK. The launch of the National Child Mortality Database for England provides a mechanism to analyse national trends in the circumstances around CYP deaths and identify patterns in modifiable factors. Establishing similar unified data systems for CYP mortality in Scotland, Wales and Northern Ireland should be a priority. Finally, continuing to monitor the UK's progress compared with other high-income countries will provide accountability and a powerful incentive to improve outcomes. ${ }^{5}$

\section{CONCLUSIONS}

We found UK mortality among CYP to be higher than a comparable group of high-income countries for common infections and multiple NCD causes. There is an urgent need for further analyses to assess how social, economic and health system determinants to survival in the UK contribute to excess CYP mortality, andfactors specific to the causes of death we have identified.

\section{Twitter Joseph Lloyd Ward @_joe_ward}

Contributors All authors conceptualised the paper. JLW and RMV planned the analyses, and JLW downloaded the data and undertook the analysis. All authors contributed to interpretation of the results. JLW led the writing of the paper with contributions from RMV and IW.

Funding JLW is funded by the Medical Research Council. RMV and IW received no specific funding for this analysis.

Competing interests None declared.

Patient consent for publication Not required.

Ethics approval Ethical approval was not required for this analysis of publicly available data.

Provenance and peer review Not commissioned; externally peer reviewed.

Data availability statement Data are publicly available from the WHO World Mortality Database (http://www.who.int/healthinfo/mortality_data/en/).

ORCID iDs

Joseph Lloyd Ward http://orcid.org/0000-0001-7263-8845

Russell M Viner http://orcid.org/0000-0003-3047-2247

\section{REFERENCES}

1 Viner R. State of child health: report 2017. London: Royal College of Paediatrics \& Child Health, 2017

2 Wolfe I, Macfarlane A, Donkin A, et al. Why children die: death in infants, children and young people in the UK. Part A. Royal College of Paediatrics and Child Health 2014.

3 Wolfe I, Donkin A, Marmot M, et al. Uk child survival in a European context: recommendations for a national countdown collaboration. Arch Dis Child 2015;100:907-14

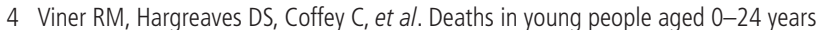
in the UK compared with the EU15+ countries, 1970-2008: analysis of the who mortality database. The Lancet 2014:384:880-92.
5 Viner RM, Ward JL, Wolfe I. Countdown for UK child survival 2017: mortality progress and targets. Arch Dis Child 2018;103:184-479.

6 Zylbersztejn A, Gilbert R, Hjern A, et al. Child mortality in England compared with Sweden: a birth cohort study. Lancet 2018;391:2008-18.

7 Euro-Peristat Project. European perinatal health report. core indicators of the health and care of pregnant women and babies in Europe in 2015, 2018. Available: www. europeristat.com

8 Wang $\mathrm{H}$, Abajobir AA, Abate $\mathrm{KH}$, et al. Global, regional, and national under-5 mortality, adult mortality, age-specific mortality, and life expectancy, 1970-2016: a systematic analysis for the global burden of disease study 2016. The Lancet 2017:390:1084-150

9 Naghavi M, Abajobir AA, Abbafati C, et al. Global, regional, and national age-sex specific mortality for 264 causes of death, 1980-2016: a systematic analysis for the global burden of disease study 2016. The Lancet 2017;390:1151-210.

10 Tambe P, Sammons HM, Choonara I. Why do young children die in the UK? A comparison with Sweden. Arch Dis Child 2015;100:59-931.

11 Tambe P, Sammons HM, Choonara I. Child mortality of children aged 5-15 years in the UK and Sweden: a comparison. Archives of disease in childhood 2016:archdischild-2015-310109.

12 Wolfe I, Thompson M, Gill P, et al. Health services for children in Western Europe. The Lancet 2013;381:1224-34.

13 Sidebotham $\mathrm{P}$, Fraser J, Fleming $\mathrm{P}$, et al. Patterns of child death in England and Wales. Lancet 2014;384:904-14.

14 Hardelid P, Dattani N, Cortina-Borja M, et al. Contribution of respiratory tract infections to child deaths: a data linkage study. BMC Public Health 2014;14:1191.

15 Zylbersztejn A, Gilbert R, Hjern A, et al. Origins of disparities in preventable child mortality in England and Sweden: a birth cohort study. Arch Dis Child 2020;105:53-61.

16 Murray CJL, Richards MA, Newton JN, et al. Uk health performance: findings of the global burden of disease study 2010. Lancet 2013;381:997-1020.

17 Hardelid P, Gilbert R. Accurate data on all injury deaths is vital for monitoring suicide prevention: BMJ publishing group LTD 2013.

18 Hawton K, Saunders KEA, O'Connor RC. Self-Harm and suicide in adolescents. The Lancet 2012:379:2373-82.

19 Ladhani S, Pebody RG, Ramsay ME, et al. Continuing impact of infectious diseases on childhood deaths in England and Wales, 2003-2005. Pediatr Infect Dis J 2010;29:310-3.

20 Marmot M, Marmot M. Fair Society, healthy lives: the Marmot review. strategic review of health inequalities in England post 20102010

21 Hardelid P, Dattani N, Davey J, et al. Overview of child deaths in the four UK countries. Royal College of Paediatrics and Child Health: Child Health Reviews-UK London, 2013.

22 Hood A, Waters T. Living Standards, poverty and inequality in the UK: 2016-2017 to 2021-2022: IFS report 2017

23 Smith NA, Phillips D, Simpson P, et al. A Time of Revolution? British Local Government Finance in the 2010s. IFS Report, No R121, ISBN 978-1-911102-24-3. London: Institute for Fiscal Studies (IFS), 2016.

24 Department of Health and Social Care. Public health ring-fenced grant 2019/2020 circular 2018.

25 Levy M, Andrews R, Buckingham R, et al. Why asthma still kills: the National review of asthma deaths (NRAD): Royal College of Physcians 2014.

26 Sidebotham P, Hunter L, Appleton R, et al. Deaths in children with epilepsies: a UKwide study. Seizure 2015;30:113-9.

27 Department for Education. Child death reviews: year ending 31 March 2017 2017.

28 Gianino $\mathrm{MM}$, Lenzi J, Bonaudo $\mathrm{M}$, et al. Patterns of amenable child mortality over time in 34 member countries of the organisation for economic co-operation and development (OECD): evidence from a 15-year time trend analysis (2001-2015). BMJ Open 2019;9:e027909.

29 NHS England. The NHS long term plan 2019.

30 Schneider EC. Mirror mirror 2017: international comparison reflects flaws and opportunities for better US health care: Commonwealth fund 2017.

31 Wolfe I, Mandeville K, Harrison K, et al. Child survival in England: strengthening governance for health. Health Policy 2017;121:1131-8.

32 Pearson G. Why children die: the report of a pilot Confidential enquiry into child death by CEMACH (Confidential enquiry into maternal and child health). Clin Risk 2008;14:166-8. 